\title{
Widespread use of anti-retroviral therapy elevated the prevalence of dyslipidemia in HIV-infected Chinese patients
}

\author{
He Ye, Yu Min Hong ${ }^{\circledR}$, Song Xiao Jing,Yang Hui, Zhang Xiao Juan, Zhao Yan Wei* \\ Department of internal medicine, Peking Union Medical College Hospital Chinese \\ Academy of Medical Sciences Peking Union Medical College, Beijing, China
}

\begin{abstract}
With the widespread use of high-efficiency antiretroviral therapy (HAART), the life expectancy of HIV-infected patients has been significantly extended. However, the metabolic complications among HIV-infected patients treated with HAART have become the most common problem in the world. It is very important to explore the incidence of dyslipidaemia and studies on the role of potential risk factors in HIV-infected Chinese patients treated with HAART are sparse. Therefore, we designed current study, to investigate the effects of therapeutic intervention and continuous information support on the lifestyle of HIV/AIDS patients with dyslipidaemia. Three hundred and six HIV/AIDS patients admitted to the AIDS clinic in Beijing from January 2016 to January 2017 were recruited and assigned into two groups: the treatment group $(n=64)$ and the control group $(n=64)$. The median age of the participants was $38.8 \pm 11.0$ years (range $20-75$ years). The prevalence of dyslipidemia in control and treatment group was (59/64) $92.2 \%$ and (53/64) $82.8 \%$, respectively. In this study, low HDLcholesterol (HDLC) led to abnormalities 47/64 (73.3\%) in the control group and 35/64 (54.7\%) in HAART-treatment group. Additionally, HAART group showed higher triglyceride, total cholesterol, and low-density lipoprotein $(246.1 \pm 171.8,1.73 \pm 1.61 \mathrm{mmol} / \mathrm{L}, 4.46 \pm 1.1 \mathrm{mmol} / \mathrm{L}, 2.54 \pm 0.74 \mathrm{mmol} / \mathrm{L})$. In multivariate analysis, gender, marital status, high BMI, dietary habits and physical activity were potential risk factors for dyslipidemia in HIV-infected Chinese patients. In this study, we reported high prevalence dyslipidemiain two HIV infected groups. We suggest that the appropriate diagnosis should be performed for analyzing the metabolic complications in HIV-infected Chinese patients. Further studies are very important to understand the role of potential risk factors in metabolic complications.
\end{abstract}

Keywords: Metabolic Complications; Lipid; Dyslipidaemia; HIV; HAART

\section{INTRODUCTION}

AIDS is an infectious disease characterized by immunodeficiency caused by human immunodeficiency virus (HIV) (Brooks, Buchacz, Gebo, Mermin, 2012). The HIV/AIDS epidemic continues to pose a humanitarian crisis to the world's most disadvantaged communities. Today, 36.9 million people are infected with HIV, $70 \%$ of who reside in sub-Saharan Africa.

*Correspondence: Z. Y. Wei. Department of internal medicine. Peking Union Medical College Hospital Chinese Academy of Medical Sciences Peking Union Medical College. Beijing, China, 100730. E-mail: zhaoyw2486@163.com
Antiretroviral therapy (ART) confers those adherents to the lifelong drug regimen near normal life expectancy. According to statistics from China CDC, as of 2015, there were about 580,000 HIV/AIDS patients in China. The main treatment for AIDS is high-efficiency antiretroviral therapy (HAART). Daily HAART treatment can significantly inhibit HIV replication and reduce viral invasion, thus greatly prolonging the lifespan of HIV/ AIDS patients (Grinsztejn et al., 2013; Lynch, Clark, Zhanel, (2013). However, long-term HAART treatment also brings a series of metabolic abnormalities to HIV/ AIDS patients. Hyperlipidemia is one of the most common manifestations, as well as one of the important 
risk factors for secondary disease in HIV/AIDS patients according to study findings. Therefore, attention to abnormal lipid metabolism in HIV/AIDS patients is of immense significance to improve the quality of life of this particular population.

There are different studies documented on the metabolic complication (hyperlipidemia) in HIV/AIDS patients treated with HAART in the world (Dave et al., 2011; Oh et al., 2017), most of which have been reported in developed countries and areas such the US and Europe (Dave et al., 2011). These data cannot be extrapolated to developing countries like China, which bear the major HIV burden. While HAAT-instigated CD4 counts are increasing, wealthy patients initiate HAART at the complex phase of HIV infection. In developing countries, antiretroviral drugs are correlated with elevated menace of dyslipidaemia, such as stavudine (d4T) andzidovudine, and are still extensively prescribed. Differences in potential risk factor such genetic background, age, education, diet, and living habits may also involve in lipid responses to HAART. Therefore, it is very important to explore the effects of HAART treatment on metabolic complications and highlight its potential risk factors.

To date, different research groups have explored the metabolic complications in HIV-infected patients in China, but such investigations were limited to specific areas. In addition, these studies did not comprehensively analyze potential risk factors. Therefore, we designed this study to investigate the prevalence of metabolic complications in HIV-infected Chinese patients and highlight the role of potential risk factors for the occurrence of metabolic complications in HIV-infected Chinese patients.

\section{METHODS}

\section{Participants}

In this study, a total of 128 HIV/AIDS patients with dyslipidemia were recruited in the three AIDS clinics in Beijing. The standard of AIDS diagnosis refers to the "AIDS treatment guidelines" formulated in 2006; the diagnostic criteria for hyperlipidemia refer to the "Guidelines for the Prevention and Treatment of Chinese Adult Blood Lipids"; the average age of participants is 38.7 years. All participants were randomly divided into three groups: 1). Treatment lifestyle combined with continuous information support group, 2). Therapeutic lifestyle adjustment group, and $3)$. Regular group. A participant was qualified for the study if he/she: (1) was above the age of 18 years, (2) was under ART treatment, (3) had a dyslipidic edge state (one or more of the following): (i) Total cholesterol (TC) $\geq 5.2$ (200) and $<6.2$ (240), (ii) LDL-C) $\geq 3.4$ (130) and $<4.1$ (160), (iii) Triglycerides (TG) $\geq 1.7$ (150) and $<2.3$ (200), (4) voluntarily agreed to sign the patient's informed consent and guaranteed follow-ups, (5) was a permanent resident of local area during the experiment. Similarly, a participant was disqualified if (1) he / she has been currently diagnosed with opportunistic infections (specified by the National AIDS Treatment Guidelines) or AIDS-related malignant tumors at the time of enrollment; or opportunistic infections occurred within 3 months before the enrollment, and the condition remained unstable for 2 weeks before the enrollment, (2) he/she had a history of diabetes and hypertension, (3) overweight (BMI>24), (4) has not received lipidlowering drug treatment for nearly 1 week.

\section{Ethical statement}

The experimental protocol was permitted by local Institutional Review Board of concerned hospitals. All participants agreed and provided their consent.

\section{Data collection}

A questionnaire was typically developed for this study. Participants attended the study visit after fasting overnight. All participants were interviewed by a trained interviewer in local dialect of Chinese by using a standardized questionnaire to elicit information on the alcohol consumption, smoking, and current medication. We also collected details about HAART, CD4 counts, HIV viral load and opportunistic infections. Additionally, a trained nurse also measured participants' weight, height, waist-hip ratio (WHR) and waist circumference.

\section{Biochemical analysis}

All samples were collected and centrifuged to isolate serum and serum was stored at $-80{ }^{\circ} \mathrm{C}$ until further analysis. Triglycerides and Total cholesterol (TC) were calculated by enzymatic colorimetry on a Cobas 6000 autoanalyzer (Roche, Switzerland). At the same time, HDL cholesterol (HDLC) was calculated through the widely used direct method (Roche HDLC3 assay, 
Switzerland). LDL cholesterol (LDLC) was measured using the Friedewald formula.

\section{Classification of dyslipidemia}

In this study, we used the National Cholesterol Education Program Adult Treatment Panel III (NCEP ATP-III) protocol to classify lipid abnormalities (Jama. 2001; 285(19):2486-97). We considered TC, LDLC and triglycerides of $>5.2 \mathrm{mmol} / \mathrm{L},>2.5 \mathrm{mmol} / \mathrm{L}$ and $>1.7$ $\mathrm{mmol} / \mathrm{L}$, respectively, as elevated and HDLC of $<1.0$ $\mathrm{mmol} / \mathrm{L}$ as low, irrespective of gender. Participants with at least one abnormal lipid parameter were classified as dyslipidemia.

\section{Statistical analysis}

In this study, we expressed continuous variables as mean or median (quartile range) and we used Student's t-test to compare normal distributions. We used the Mann-Whitney $U$ test to perform a skewed distribution of continuous variables. However, $\chi_{2}$ test was used to compare categorical variables. Firstly, we performed univariate analysis; only variables with a $P$ value of less than 0.05 in the univariate analysis were included in the logistic regression model for multivariate analysis. IBM SPSS Statistics 20.0 was used for all the statistical analysis. $\mathrm{P}<0.05$ was considered to indicate statistical significance.

\section{RESULTS}

\section{Demographic variables}

All participants were recruited from three HIV clinics in Beijing, China (Table I). The median age of the participants was $38.8 \pm 11.0$ years (range $20-75$ years) (mean $=38$ years, $\mathrm{SD}=11.0$ years). $90 \%$ of the totals were men and $10 \%$ of the totals were women. In addition, $51 \%$ of the participants came from rural areas, and $49 \%$ were from urban areas, of which, $7 \%$ attended primary school, $20 \%$ middle school, $20 \%$ secondary school and $53 \%$ received university level education. $64 \%$ of the participants were drinkers and $38 \%$ were smokers. Obesity (BMI_30 $\mathrm{kg} / \mathrm{m}^{2}$ ) was more common than underweight and was found in 44/128 (34\%) participants. A summary of the participants' characteristics is shown in Table I.
TABLE I - Demographic characteristics of participants'

\begin{tabular}{lcc}
\hline Variables & Participants & Percentage \\
\hline Grouping & 64 & 50 \\
Control & 64 & 50 \\
Treatment group & & \\
\hline Gender & 115 & 89.8 \\
Men & 13 & 10.2 \\
Women & & \\
\hline Nation & 120 & 93.7 \\
Han Chinese & 8 & 6.3 \\
\hline
\end{tabular}

\section{Body Mass index(BMI)}

Underweight $<18$

6

Normal weight $18-24$

78

60.9

Overweight 24-28

33

25.8

Obesity $>28$

10

7.8

\section{Education}

Primary 10

Middle

Secondary

25

19.5

Bachelor

67

52.3

\section{Employment status}

Unemployed 19 14.8

Self-employed 39

30.5

Private employed

70 
TABLE I - Demographic characteristics of participants'

\begin{tabular}{lcc}
\hline Variables & Participants & Percentage \\
\hline Job category & 24 & 18.7 \\
Labor & 104 & 81.3 \\
Managerial & & \\
\hline Marital status & 83 & 64.8 \\
Single & 45 & 35.2 \\
Married & & \\
\hline Income & 43 & 33.6 \\
Low income & 76 & 59.4 \\
Middle income & 9 & 7.0 \\
High income & 56 & 63.7 \\
\hline Do you smoke & 43 & 56.3 \\
No & & \\
Yes & & \\
\hline Do you drink & & \\
No & & \\
Regular & & \\
\hline & & \\
\hline
\end{tabular}

\section{Lipids Analysis}

In this study, the baseline lipid values have been shown in Table II. We classified(59/64) $92.2 \%$ and $(52 / 64) 81.2 \%$ of the control group and HAART treatment group participants diagnosed with dyslipidemia by using the definition given in the Methods section. In this study, low HDLC $(<1.0 \mathrm{mmol} / \mathrm{L})$ was one of the largest lipid abnormalities, found in $68.7 \%(44 / 64)$ and $46.9 \%(30 / 64)$ of the control group and HAART treatment group, respectively. Hypertriglyceridemia of $>5.0 \mathrm{mmol} / \mathrm{L}$ was detected in $1.5 \%(1 / 64)$ of the control group and $6.2 \%(4 / 64)$ of the treatment group, whereas hypercholesterolemia of $>10 \mathrm{mmol} / \mathrm{L}$ was found in $3.1 \%(2 / 64)$ of the control group and $1.1 \%(1 / 64)$ of the treatment group. LDL hypercholesterolaemia of $>4.9 \mathrm{mmol} / \mathrm{L}$ was found in $1.6 \%(1 / 64)$ of the control group and $4.6 \%(3 / 64)$ of the treatment group. Severe hypercholesterolaemia, $(\mathrm{TC}>7.5 \mathrm{mmol} / \mathrm{L})$, was detected in $0 \%(0 / 64)$ of the control group and $16 \%(10 / 64)$ of the treatment group, respectively.

TABLE II - Baseline lipid values in control and treatment group

\begin{tabular}{lcc}
\hline Variable & $\begin{array}{c}\text { Control } \\
\mathbf{n}=\mathbf{6 4}\end{array}$ & $\begin{array}{c}\text { Treatment } \\
\text { group } \\
\mathbf{n = 6 4}\end{array}$ \\
\hline $\begin{array}{l}\text { Total cholesterol } \\
(\mathrm{mmol} / \mathrm{L})^{*}\end{array}$ & $4.1(3.6,4.4)$ & $4.36(3.81-4.97)$ \\
$\begin{array}{l}\text { Triglycerides } \\
(\mathrm{mmol} / \mathrm{L})^{*}\end{array}$ & $1.1(0.85,1.25)$ & $1.28(0.86-2.05$ \\
HDLC $(\mathrm{mmol} / \mathrm{L})^{*}$ & $0.90(0.75,1.05)$ & $1.06(0.91-1.23)$ \\
LDLC $(\mathrm{mmol} / \mathrm{L})^{*}$ & $2.32(1.93,2.81)$ & $2.5(2.04-2.93)$ \\
$\mathrm{CD} 4 \mathrm{count}$ & $237(113-346.75)$ & $407(276-498)$ \\
\hline
\end{tabular}

\section{Potential risk factors}

To investigate the potential risk factors for dyslipidemia in Chinese HIV-infected patients, we performed univariate (Table III) and multivariate analysis (Table IV). We found that men were more prone to dyslipidemia $((\mathrm{P}=0.047, \mathrm{OR}=0.162)$. Similarly, married participants were more prone to dyslipidemia $((\mathrm{P}=0.045, \mathrm{OR}=2.263)$. Aged participants significantly suffered more dyslipidemia $\{(<30$ years' old $\mathrm{P}=0.019)$, (30-39 years' old $\mathrm{P}=0.010, \mathrm{OR}=33.705),(40-49$ years' old, $\mathrm{P}=0.060, \mathrm{OR}=41.996)\}$. But in the case of $\mathrm{BMI}$, participants with higher BMI were more prone to dyslipidemia $\quad\{((\mathrm{BMI}<18, \quad \mathrm{P}=0.013), \quad$ overweight (BMI $=24-28, \quad \mathrm{P}=0.003, \quad \mathrm{OR}=155.331), \quad$ obesity $(\mathrm{BMI}>28, \mathrm{P}=0.059, \mathrm{OR}=29.434)$ ). We also investigated the effect of eating habits on dyslipidemia, and we found that participants with lower comprehensive score were more likely to have dyslipidemia $(\mathrm{P}=0.032$, $\mathrm{OR}=0.044)$. When we compared the dietary habit with 
gender, we found that women's blood lipid levels were more relevant to diet as compared to men $(\mathrm{P}=0.020$, $\mathrm{OR}=21.344)$ (Table IV).

TABLE III - Univariate analysis of potential risk factor for metabolic abnormalities

\begin{tabular}{lc}
\hline Variables & $P^{*}$ \\
\hline Education & 0.022 \\
employment status & $0.055,0.026$ \\
Dietary habits & $\mathbf{0 . 0 2 9}$ \\
marital status & $<0.001$ \\
$\begin{array}{l}\text { Antiviral treatment } \\
\text { drug category }\end{array}$ & 0.035 \\
generation & $0.004,0.001$ \\
BMI index & $<0.001,0.054$ \\
Course/month & $0.018,0.002$ \\
Antiviral time & 0.016 \\
\hline
\end{tabular}

TABLE IV - Multiple logistic regression analysis of potential risk factor for metabolic abnormalities

\begin{tabular}{lcc}
\hline Variable s & $P^{*}$ & OR \\
\hline Gender & 0.047 & 0.162 \\
marital status & 0.045 & 2.263 \\
Age group & 0.019 & \\
Age group(1) & 0.010 & 33.705 \\
Age group(2) & 0.060 & 41.996 \\
BMi group 1 & 0.013 & \\
BMI group 2 & 0.003 & 155.331 \\
\hline
\end{tabular}

TABLE IV - Multiple logistic regression analysis of potential risk factor for metabolic abnormalities

\begin{tabular}{lcc}
\hline $\begin{array}{l}\text { Variable s } \\
\text { Dietary habits group(1) }\end{array}$ & $P^{*}$ & OR \\
\hline $\begin{array}{l}\text { Gender by eating habits } \\
\text { comprehensive score group(1) }\end{array}$ & 0.032 & 0.044 \\
$\begin{array}{l}\text { BMI index (2) by physical } \\
\text { activity level (1) }\end{array}$ & 21.344 \\
$\begin{array}{l}\text { BMI index (2) by physical } \\
\text { activity level (2) }\end{array}$ & 0.034 & 0.045 \\
$\begin{array}{l}\text { BMI Grading (3) by Physical } \\
\text { Activity Level (2) }\end{array}$ & 0.005 & 0.015 \\
\hline
\end{tabular}

\section{DISCUSSION}

Due to the widespread use of HAART treatment, the life expectancy of HIV-infected patients is continually increasing, but metabolic complications caused much concern in those cases. In this study, firstly, we assessed the prevalence of metabolic complications in HIV-infected Chinese patients, and further we explored the possible role of potential risk factors for the occurrence of metabolic complications in HIV-infected Chinese patients.

In this study, prevalence of dyslipidaemia was very high in both control and treatment groups, with low HDLC as the most common abnormality. Additionally, we found elevated HDLC levels in HAART participants. In this study, we defined triglycerides $>5.0 \mathrm{mmol} / \mathrm{L}$ or dyslipidaemia, as LDLC $>4.9 \mathrm{mmol} / \mathrm{l}$. In this study, we observed very high prevalence of dysglycaemia in HAART treatment group than the control group. Our observations are completely in line with previously reported data (Dave et al., 2011). Additionally, linkage between lipid abnormalities and HIV has already been documented (Dave et al., 2016; Grunfeld et al., 1989; Grunfeld et al., 1991). In many studies that have already been reported, HIV disease is linked with decreased concentrations of TC, LDLC, and HDLC, whereas hypertriglyceridaemia has a positive correlation with lipid abnormalities and the degree of immune-suppression. Chronic infection occurred due 
to continued acute phase reaction, which is usually associated with elevated triglycerides due to the decline in lipoprotein lipase activity. It has been suggested that the boost in triglyceride-rich lipoproteins potentially promotes the substitution of triglycerides into HDL by hepatic lipase after lipolysis, accounting for lower HDLC concentration (van der Voort et al., 2003;van der Westhuyzen, de Beer, Webb, 2007). In this study, we found high lipid abnormalities are consistent with the previously reported observation (Jantarapakde et al., 2014; Muhammad, Sani, Okeahialam, 2013; Oka et al., 2012). It has already been highlighted that immunosuppression increased with the decrease of CD4 counts, which is linked with an increase in triglycerides. Additionally, the risk of obesity increased with the increase of waist-hip ratio (WHR), which is also linked with higher triglycerides figures. Another important observation of the current study was low HDLC in the control group, which is completely consistent with previously reported studies (Asiki et al., 2015; Bekolo, Nguena, Ewane, et al., Kollo, 2014; Kuti et al., 2015; Seedat, Mayet, Latiff, Joubert, 1992). Many new treatment trends have been adopted over the last decade. Therefore, the characteristics of HIV-infected patients have been distorted. When, we compared the metabolic complication between control and treatment groups by using antiretroviral drugs (HAART), median HDL-cholesterol, total cholesterol, and triglyceride levels elevated in the treatment group. Additionally, the prevalence of dyslipidemia and lipodystrophy also significantly elevated in the treatment group. Our study observations are completely consistent with previous study findings (Jantarapakde et al., 2014). Jantarapakde, a Thailand based scientist has reported significantly higher prevalence of lipodystrophy in HIV patients treated with HART. Similarly, the mean of HDL-cholesterol, total cholesterol and triglyceride levels also increased in other investigations (Gupta, Biswas, Sharma, 2011; Wu et al., 2012). In view of abovementioned facts, we can suggest that antiretroviral therapy may influence the incidence of metabolic complications.

In this study, we performed univariate and multivariate analysis to explore the role of potential risk factors in the prevalence of metabolic complication. We found that gender, BMI, marital status, age, dietary habits and physical activity levels were significant risk factors for dyslipidemia. It has already been reported by Ishikawa-Takataet al. that BMI was associated with the prevalence of dyslipidemia in Japanese than Caucasian, despite of a low BMI. These observations are supported by data (Ishikawa-Takata, Ohta, Moritaki, et al., 2002. Other investigations have reported the correlation between PI exposure and metabolic complications (Gazzaruso et al., 2002; Samaras et al., 2007).But in this study, multivariate analysis showed non-significant differences. Moreover, dyslipidemia may be correlated with lipodystrophy in HIV-infected patients, thus inducing fact accretion (Wohl et al., 2008).

Our study has several important strengths and limitations. To the best of our knowledge, here for the first time, we have reported in detail the metabolic effects of HART on Chinese HIV infected patients. All participants took part in an inclusive and complete metabolic evaluation at the time of recruitment. Additionally, in this study, we have explored the effects of potential risk factors on hypolipidemia. Small number of participants is the only limitation of the current study.

In conclusion, we found that participants in HART treatment group had higher triglycerides, TC, LDLC and HDLC than those in the control group. HART is significantly associated with an increase in dyslipidemia. Based on these findings, we suggest that there exists a correlation between smoking and another risk factor such as age, smoking, BMI, gender, marital status and dietary habits. We will attempt to explain the correlations of potential risk factors in our future study. Thus, based on our observations, car should be taken during the treatment of HIV infected patients. Therefore, we suggest the importance of discovering a new drug and it is mandatory for the treatment of HIV infected patients. Furthermore, considering the future large scale, multi-ethnic population-based study must be conducted to obtain comprehensive information on the prevalence of metabolic complication in various HIV infected Chinese patients.

\section{DECLARATIONS}

\section{Availability of data and materials}

The datasets generated or analyzed during the current study are available from the corresponding authors upon reasonable request.

\section{Competing interests}

The authors declare that they have no competing interests. 


\section{Consent for publication}

Not applicable.

\section{Ethics approval and consent to participate}

The study was approved by the ethics committees of the ${ }^{1}$ Department of internal medicine, Peking Union Medical College Hospital Chinese Academy of Medical Sciences Peking Union Medical College, Beijing, China. Informed consent was obtained from all individual participants included in the study.

\section{Authors' contributions}

He Ye and Yu MinHong wrote and revised the draft manuscript and subsequent manuscripts, and analyzed participants' samples. Zhao YanWei designed the study, revised the draft manuscript. Song XiaoJing, Yang Hui and Zhang XiaoJuanconceived and designed the study, appraised relevant studies and assisted in drafting and revising the manuscript. All authors read and approved the final manuscript.

\section{ACKNOWLEDGMENTS}

We are grateful to the participants, primary care doctors, and nurses who participated in this survey. Working spaces, facilities and staffs were provided by Department of Internal Medicine, Peking Union Medical College Hospital Chinese Academy of Medical Sciences Peking Union Medical College, Beijing, China.

\section{REFERENCES}

Asiki G, Murphy GA, Baisley K, Nsubuga RN, Karabarinde A, Newton R, Sandhu MS. Prevalence of dyslipidaemia and associated risk factors in a rural population in SouthWestern Uganda: a community based survey. PLoS One. 2015;10(5):e0126166.

Bekolo CE, Nguena MB, Ewane L, Bekoule PS, Kollo B. The lipid profile of $\mathrm{HIV}$-infected patients receiving antiretroviral therapy in a rural Cameroonian population.BMC Public Health. 2014;14:236.

Brooks JT, Buchacz K, Gebo KA, Mermin J. HIV infection and older Americans: the public health perspective. Am J Public Health. 2012;102(8):1516-1526.
Dave JA, Lambert EV, Badri M, West S, Maartens G, Levitt NS. Effect of nonnucleoside reverse transcriptase inhibitorbased antiretroviral therapy on dysglycemia and insulin sensitivity in South African HIV-infected patients.J Acquir Immune DeficSyndr. 2011;57(4):284-289.

Dave JA, Levitt NS, Ross IL, Lacerda M, Maartens G, Blom D. Anti-Retroviral Therapy Increases the Prevalence of Dyslipidemia in South African HIV-Infected Patients. PLoS One. 2016;11(3):e0151911.

Gazzaruso C, Sacchi P, Garzaniti A, Fratino P, Bruno R, Filice G. Prevalence of metabolic syndrome among HIV patients. Diabetes Care. 2002;25(7):1253-1254.

Grinsztejn B, Luz PM, Pacheco AG, Santos DV, Velasque L, Moreira RI, Veloso VG (2013). Changing mortality profile among HIV-infected patients in Rio de Janeiro, Brazil: shifting from AIDS to non-AIDS related conditions in the HAART era. PLoSOne. 2013;8(4):e59768.

Grunfeld C, Kotler DP, Hamadeh R, Tierney A, Wang J, Pierson RN. Hypertriglyceridemia in the acquired immunodeficiency syndrome. Am J Med. 1989;86(1):27-31.

Grunfeld C, Kotler DP, Shigenaga JK, Doerrler W, Tierney A, Wang J, Feingold KR. Circulating interferon-alpha levels and hypertriglyceridemia in the acquired immunodeficiency syndrome. Am J Med. 1991;90(2):154-162.

Gupta V, Biswas A, Sharma SK. Metabolic and body composition changes after six months of highly active antiretroviral therapy in northern Indian patients. Int $\mathrm{J}$ STDAIDS.2011;22(1), 46-49.

Ishikawa-Takata K, Ohta T, Moritaki K, Gotou T, Inoue S. Obesity, weight change and risks for hypertension, diabetes and hypercholesterolemia in Japanese men. Eur JClinNutr. 2002;56(7):601-607.

Jantarapakde J, Phanuphak N, Chaturawit C, Pengnonyang S, Mathajittiphan P, Takamtha P, Phanuphak P. Prevalence of metabolic syndrome among antiretroviral-naive and antiretroviral-experienced HIV-1 infected Thai adults. AIDS Patient Care STDS, 2014;28(7):331-340.

Kuti M A, Adesina OA, Awolude OA, Ogunbosi BO, Fayemiwo SA, Akinyemi JO, Adewole IF. Dyslipidemia in ART-Naive HIV-Infected Persons in Nigeria--Implications for Care. J IntAssocProvid AIDS Care. 2015;14(4):355-359.

Lynch JP, Clark NM, Zhanel GG. Evolution of antimicrobial resistance among Enterobacteriaceae (focus on extended spectrum beta-lactamases and carbapenemases).Expert OpinPharmacother. 2013;14(2):199-210. 
He Ye, Yu Min Hong, Song Xiao Jing,Yang Hui, Zhang Xiao Juan, Zhao Yan Wei

Muhammad S, Sani MU, Okeahialam BN. Prevalence of dyslipidemia among human immunodeficiency virus infected Nigerians. Ann Afr Med. 2013;12(1):24-28.

Oh DH, Ahn JY, Kim SI, Kim MJ, Woo JH, Kim WJ, Korea HIV. Aids Cohort Study. Metabolic Complications among Korean Patients with HIV Infection: The Korea HIV/AIDS Cohort Study. J Korean Med Sci. 2017;32(8):1268-1274.

Oka F, Naito T, Oike M, Imai R, Saita M, Inui A, Shimbo T. Correlation between HIV disease and lipid metabolism in antiretroviral-naive HIV-infected patients in Japan. J Infect Chemother. 2012;18(1):17-21.

Samaras K, Wand H, Law M, Emery S, Cooper D, Carr A. Prevalence of metabolic syndrome in HIV-infected patients receiving highly active antiretroviral therapy using International Diabetes Foundation and Adult Treatment Panel III criteria: associations with insulin resistance, disturbed body fat compartmentalization, elevated C-reactive protein, and [corrected] hypoadiponectinemia. Diabetes Care. 2007;30(1):113-119.

Seedat YK, Mayet FG, Latiff GH, Joubert G. Risk factors and coronary heart disease in Durban blacks--the missing links. S Afr Med J. 1992;82(4):251-256.

van der Voort PHJ, Gerritsen RT, Bakker AJ, Boerma EC, Kuiper MA, de Heide L. HDL-cholesterol level and cortisol response to synacthen in critically ill patients. Intensive Care Med. 2003;29(12):2199-2203.

van der Westhuyzen, DR, de Beer FC, Webb NR. HDL cholesterol transport during inflammation. CurrOpinLipidol. 2007;18(2):147-151.

Wohl D, Scherzer R, Heymsfield S, Simberkoff M, Sidney $\mathrm{S}$, Bacchetti P. Investigators, FramStudy. The associations of regional adipose tissue with lipid and lipoprotein levels in HIV-infected men.J Acquir Immune DeficSyndr. 2008;48(1):44-52.

Wu PY, Hung CC, Liu WC, Hsieh CY, Sun HY, Lu CL, Chien KL. Metabolic syndrome among HIV-infected Taiwanese patients in the era of highly active antiretroviral therapy: prevalence and associated factors. J Antimicrob Chemother. 2012;67(4):1001-1009.

Received for publication on $23^{\text {rd }}$ October 2018 Accepted for publication on $15^{\text {th }}$ February 2019 